\title{
Bioanalysis
}

\section{Best practices for discovery bioanalysis: balancing data quality and productivity}

\author{
"In summary, data quality is as important as speed. The true challenge for \\ discovery bioanalysis is to balance the needs for quality and speed and to deliver \\ high quality data quickly."
}

\begin{abstract}
Keywords: bioanalysis $\bullet$ data quality $\bullet$ discovery bioanalysis $\bullet$ matrix effects $\bullet$ preemptive investigation $\bullet$ productivity $\bullet$ regulated bioanalysis
\end{abstract}

Frequently, the term 'fast turnaround' is the first thing that comes to mind for discovery bioanalysis. During drug discovery, speed is essential for in vitro and/or in vivo screening of large numbers of compounds. In the recent 10-15 years, there has been much interest, effort and investment in optimizing workflow and implementing informatics solutions to increase productivity and to improve turnaround time [1-6]. The results have been impressive. It is now possible to perform in vitro assays for hundreds of compounds within a week with just three or four full-time personnel. Furthermore, the discovery PK bioanalysis turnaround time is usually 5 days or less [4].

By contrast, data quality has received less attention. The time and effort spent developing discovery bioanalysis methods is limited, which has adversely affected data quality. The word 'discovery' is even being used sometimes for "questionable data". Although this risk is widely accepted for compound screening, selection, and optimization purpose, it has raised concerns for data usability. Too often, it has led to project teams receiving conflicting discovery bioanalysis results, which require some studies to be repeated and lead to delays in project decision making. Undoubtedly, such occurrences are not desirable even though the data were generated quickly.

In summary, data quality is as important as speed. The true challenge for discovery bioanalysis is to balance the needs for quality and speed and to deliver high quality data quickly [7].

\section{Balancing data quality \& productivity}

For discovery bioanalysis, high quality data requires a reasonable amount of confidence associated with the data for drug discovery purposes. High productivity means achieving the needed confidence as quick as possible. To accomplish both, we must treat discovery bioanalysis differently from the regulated bioanalysis.

Discovery bioanalysis is not 'simplified regulated bioanalysis', that is. analysis without the method validation and compliance components and with more relaxed acceptance criteria. For regulated bioanalysis, method validity is ensured through distinct method development and method validation steps. The level of rigor exercised during method validation ensures that a validated method is not likely to behave differently over time during sample analysis. Subsequently, quality assessment during the sample analysis is usually limited to the evaluation of QC performance. Discovery bioanalysis, on the other hand, often uses a generic method to analyze samples without method validation. The analysis run serves dual purposes: method establishment and sample analysis. Therefore, the quality-by-QC approach used for regulated bioanalysis is not suitable. The data analysis for discovery bioanalysis has to be a comprehensive assessment of accuracy, matrix effects, interference, and carry-over in order to confirm method validity. In addition, sample preparation and data acquisition also require careful consideration. For

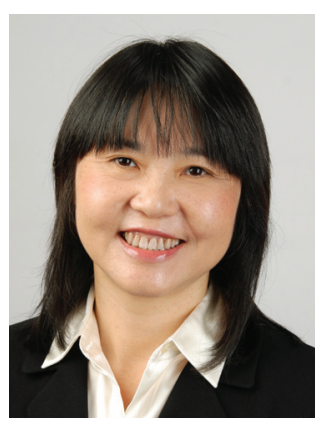

Stacy Ho

Drug Metabolism \& Pharmacokinetics (DMPK), Disposition, Saftety \& Animal Research (DSAR), Sanofi, 153 Second Avenue, Waltham, MA 02451, USA

Tel.: +1 7814343485

Fax: +1 7814663789

stacy.ho@sanofi.com 
best results, potential co-eluting peaks and formulation excipient matrix effects should be preemptively investigated.

\section{Sample preparation}

When planning for discovery bioanalysis sample preparation, careful consideration must be given to minimize potential method problems and provide options for problem identification and method optimization. Authentic matrix is highly recommended for standard preparation to normalize potential matrix effects. If surrogate matrix is used, samples should be diluted several fold by the surrogate matrix so that the matrix contribution of the sample is small, assuming analyte sensitivity is sufficient.

Two or three IS eluting at different retention times may be included in the generic method to support the analysis of a wide range of compounds [4]. It is best if the IS provides good responses in both negative and positive ionization modes under both electrospray and atmospheric pressure ionization although not necessarily by a single IS. This will enable a quick selection of IS and ionization mode to suit the quantitation needs.

A marker compound can be added post-extraction to the supernatant dilution solvent [8] as another indicator of data quality. Single blank (contains IS only) and double blank should always be prepared so that matrix interference, if present, can be observed. To save time, the early time point samples can be prepared with and without dilution to avoid thawing and re-preparing sample when the analyzed concentration of undiluted sample is over the calibration range. Finally, incorporating precautionary sample pre-treatment based chemical structures and ADME screening results may be critical for certain compounds to ensure data quality. For example, plasma samples of carboxylic acid containing compounds should be pre-treated with acid to prevent the back conversion of acyl glucuronide metabolites to the dosed compound [9].

\section{Data acquisition}

Similar to sample preparation, data acquisition conditions must be chosen carefully to minimize potential problems and to provide options for problem identification and method optimization. Optimized generic chromatographic conditions helps to reduce the matrix effects for LC-MS/MS assay [10]. Two mass transitions for the analyte may be included in the acquisition method, which will provide an alternative if matrix effects or interference is observed in one transition. For a carboxylic acid containing drug, monitoring acyl glucuronide metabolite in addition of the parent compound helps to identify potential erroneous results $[9,11-12]$. To minimize carry-over, the injection sequence should be arranged so that the samples with the same time points are injected together and samples with a lower expected concentration should be injected before those with a higher expected concentration. Although wide calibration range is desirable for reducing the need for sample dilution, it also increases the likelihood of high carry-over. The order of magnitude for the calibration curve is often depended on the autosampler used as well as the characteristics of analyte. Beside the autosampler; the column and gradient used may also contribute to carry-over and should be examined when setting up the generic chromatographic method [13].

\section{Data analysis}

During data analysis, a discovery bioanalytical method is finalized by selecting a suitable IS, mass transition, and calibration range. The process starts by examining chromatographic performance for peak shape, retention time, and resolution. An abnormally broad peak or a peak in blank samples at or close to the retention time of analyte may affect quantitation. If chromatographic performance is acceptable, then, method performance should be examined for sensitivity, linearity, accuracy, matrix effects, and carry-over, etc. A graph of IS (and marker compound) responses versus injections (Levey-Jennings chart) is a quick and easy way to visualize matrix effects. If the IS responses in standard and unknown are very different, sample specific matrix effects are indicated and the method must be further modified. If the responses of IS and marker compound are consistent throughout the run except for one or two samples, those samples may have specific matrix effects or were prepared incorrectly and should be investigated.

Data analysis should also include evaluation of results that are not related to analytical run to further build confidence in data. For PK studies, the results should show the general trend of a PK curve. Dose solution analysis results should be within the acceptance range of target concentrations. Non-conformance dose analysis results may indicate inaccurate weighing of bioanalytical stock solution since the dose solution is typically made from a separate weighing.

\section{Preemptive investigation}

Co-eluting peak and matrix effects of formulation excipients are examples of analytical issues that cannot be readily detected under the generic method condition but can significantly affect the results [14,15]. Metabolite and interference peaks with the same mass transition co-eluting with the analyte can often be identified by re-injecting one of the samples (usually the sample after $\mathrm{C}_{\max }$ for PK samples) with slower gradient. Some formulation excipients, such as Tween 80 
and PEG400, have long been reported to cause time point dependent matrix effects [15-18]. They can lead to overestimation of key PK parameters including clearance and volume of distribution by several fold [16]. A simple dilution check described by Larger, et al. [18] can be used to identify the issue, in which the earliest time point IV sample is prepared undiluted, 5 and 10 fold diluted and analyzed with the analytical batch. Matrix effects are indicated if the analyzed concentration for the diluted sample is higher than the undiluted sample. Those preemptive investigations require extra time due to additional sample preparation and injection, but they enhance data confidence significantly.

\section{Improve quality \& productivity}

High end laboratory wide solutions, such as workflow optimization, automation, and electronic notebook, are among the most effective approaches to improving productivity. An added benefit of these approaches is that they often improve data quality at the same time. However, the resource requirements of these solutions are high. In the cases of automation and electronic notebook, people with special knowledge are needed for setup and maintenance. Due to high demand for time and money [19], and lack of expertise, small laboratories generally do not enthusiastically embrace these solutions. That does not mean that there are not ways for those laboratories to improve. In fact, there are plenty of low end solutions suitable for any size operation. The use of decision tree can be an efficient way to deal with potential analytical issue and can reduce troubleshooting time. The use of simple

\section{References}

1 Zhang N, Rogers K, Gajda K, Kagel JR, Rossi DT. Integrated sample collection and handling for drug discovery bioanalysis. J. Pharm. Biomed. Anal. 23(2-3), 551-560 (2000).

2 Patel V, Leach D, Hornberger M et al. Automating bioanalytical sample analysis through enhanced system integration. sample analysis through enhanced system integration. Bioanalysis 5(13), 1649-1659 (2013).

3 Shou WZ, Zhang J. Recent development in software and automation tools for high-throughput discovery bioanalysis. Bioanalysis 4(9), 1097-1109 (2012).

4 Bateman KP, Cohen L, Emary B, Pucci V. Standardized workflows for increasing efficiency and productivity in discovery stage bioanalysis. Bioanalysis 5(14), 1783-1794 (2013).

5 Smalley J, Xin B, Olah TV. Increasing high-throughput Discovery bioanalysis using automated selected reaction monitoring compound optimization, ultra-high-pressure liquid chromatography, and single-step sample preparation workflows. Rapid Commun. Mass Spectro. 23(21), 3457-3464 (2009).
Word and Excel templates can reduce time and errors in documentation and calculation. Training people to use software effectively and to identify method issues skillfully can be surprisingly effective in improving both quality and productivity.

As the pharmaceutical industry is placing more and more emphasis on drug discovery in order to reduce time and cost for getting a new drug to the market, the need to improve quality and productivity is constant. This in turn requires us to continually examine both high and low end improvements so that discovery bioanalysis can deliver higher quality data faster. In the next $10-15$ years, technology will drive the quality and productivity improvement. More and more laboratories will gradually move away from manual preparation and paper notebooks and embrace automation and electronic records in order to stay competitive. New technologies, such as high resolution mass spectrometer [20], will provide new tools to reduce time for method setup, combat matrix effects and interference, and generate discovery data with tighter acceptance criteria.

\section{Financial \& competing interests disclosure}

The author has no relevant affiliations or financial involvement with any organization or entity with a financial interest in or financial conflict with the subject matter or materials discussed in the manuscript. This includes employment, consultancies, honoraria, stock ownership or options, expert testimony, grants or patents received or pending, or royalties.

No writing assistance was utilized in the production of this manuscript.

6 Zhang J, Vath M, Ferraro C et al. A high-speed liquid chromatography/tandem mass spectrometry platform using multiplexed multiple-injection chromatography controlled by single software and its application in discovery ADME screening. Rapid Commun. Mass Spectrom. 27(7), 731-737 (2013).

7 Xu X, Lan J, Korfmacher WA. Rapid LC/MS/MS method development for drug discovery. Anal. Chem. 77(19), 389A-394A (2005).

8 Tsefrikas V, Zhang X. Analysis of small molecule drugs in tissue. current practices. In. Tissue Analysis for Drug Development, Ho S (Ed.) Future Science Ltd, London, UK, 9-22 (2013).

9 Zhou J, Li F, Duggan JX. LC-MS bioanalysis of acyl glucuronides. In. Handbook of LC-MS Bioanalysis. Li W, Zhang J, Tse FLS (Eds). John Wiley \& Sons, Inc, Hokoben, NJ, USA, 447-460 (2013).

10 Ye Z, Tsao H, Gao H, Brummel CL. Minimizing matrix effects while preserving throughput in LC-MS/MS bioanalysis. Bioanalysis 3(14), 1587-1601 (2011).

11 Mess J-N, Beerubee E-R, Furtado M, Garofolo F. A practical approach to reduce interference due to in-source collision- 
induced dissociation of acylglucuronides in LC-MS/MS . Bioanalysis 3(15), 1741-1751 (2011).

12 Clark GT. The importance of partnership when outsourcing exploratory bioanalysis. Bioanalysis 6(6), 733-736 (2014).

13 Williams JS, Donahue SH, Gao H, Brummel CL. Universal LC-MS method for minimized carryover in a discovery bioanalytical setting. Bioanalysis 4(9), 1025-1037 (2012).

14 Li F, Ewles M, Pelzer M et al. Case studies. the impact of nonanalyte components on LC-MS/MS-based bioanalysis. strategies for identifying and overcoming matrix effects. Bioanalysis 5(19), 2409-2441 (2013).

$15 \mathrm{Xu} \mathrm{X}$, Mei H, Wang $\mathrm{S}$ et al. A study of common discovery dosing formulation components and their potential for causing time-dependent matrix effects in high-performance liquid chromatography tandem mass spectrometry assays. Rapid Commun. Mass Spectrom. 19(18), 2643-2650 (2005).

16 Schuhmacher J, Zimmer D, Tesche F, Pickard V. Matrix effects during analysis of plasma samples by electrospray and atmospheric pressure chemical ionization mass spectrometry. practical approaches to their elimination. Rapid Commun. Mass Spectrom. 17(17), 1950-1957 (2003).

17 Tong XS, Wang J, Zheng S et al. Effect of signal interference from dosing excipients on pharmacokinetic screening of drug candidates by liquid chromatography/mass spectrometry. Anal. Chem. 74(24), 6305-6313 (2002).

18 Larger PJ, Breda M, Fraier D, Hughes H, James CA. Ionsuppression effects in liquid chromatography-tandem mass spectrometry due to a formulation agent, a case study in drug discovery bioanalysis. J. Pharm. Biomed. Anal. 39(1-2), 206-216 (2005).

19 Cohen LH. Surrendering to the robot army. why we resist automation in drug discovery and development. Bioanalysis 4(9), 985-987 (2012).

20 Korfmacher W. High-resolution mass spectrometry will dramatically change our drug-discovery bioanalysis procedures. Bioanalysis 3(11), 1169-1171 (2011). 